\title{
Duodenal and jejunal Dieulafoy's lesions: optimal management
}

This article was published in the following Dove Press journal:

Clinical and Experimental Gastroenterology

7 November 2017

Number of times this article has been viewed

\author{
Tonguç Utku Yılmaz' \\ Ramazan Kozan² \\ 'Acibadem University Atakent \\ Hospital, Organ Transplantation \\ Unit, Istanbul, Turkey, ${ }^{2}$ Eren Hospital, \\ General Surgery Unit, Istanbul, Turkey
}

\begin{abstract}
Dieulafoy's lesions (DLs) are rare and cause gastrointestinal bleeding resulting from erosion of dilated submucosal vessels. The most common location for DL is the stomach, followed by duodenum. There is little information about duodenal and jejunal DLs. Challenges for diagnosis and treatment of Dieulafoy's lesions include the rare nature of the disease, asymptomatic patients, bleeding symptoms often requiring rapid diagnosis and treatment in symptomatic patients, variability in the diagnosis and treatment methods resulting from different lesion locations, and the risk of re-bleeding. For these reasons, there is no universal consensus about the diagnosis and treatment approach. There are few published case reports and case series recently published. Most duodenal DLs are not evaluated seperately in the studies, which makes it difficult to determine the optimal model. In this study, we summarize the general aspects and recent approaches used to treat duodenal DL.
\end{abstract}

Keywords: Dieulafoy's lesion, gastrointestinal bleeding, duodenum, endoscopy

\section{Introduction}

Dieulafoy's lesions (DLs) are most often located in the stomach; also, they have been detected in the duodenum. While gastric DLs are well known, there are few reports about duodenal and small intestinal lesions. Before endoscopy was used, the diagnosis and prognosis for patients with these lesions was poor, with mortality rates ranging from $23 \%$ to $79 \%{ }^{1}$ The probability of forward-viewing endoscopy failure in the diagnosis of duodenal DL, the high-risk surgery for periampullary DL, and re-bleeding risks made the treatment and the diagnosis difficult for duodenal lesions. Similarly, localization problems in jejunal DLs prompted clinicians to search for new diagnostic and treatment modalities.

Duodenal and jejunal DLs constitute $15 \%$ and $1 \%$ of all DLs, respectively. Duodenal and jejunal DLs are responsible for 3.5\% of all gastrointestinal (GI) bleedings. Unrecognized lesions and the rare nature of their occurrence make these lesions difficult to diagnose, and it is also difficult to develop a standardized treatment for patients with DLs.

Duodenal DLs are rare and they are mostly presented as case reports. In this study, we report the most recent information about the diagnosis and treatment modalities for patients with duodenal and jejunal DLs from the limited number of studies.

\section{Pathology and etiology}

DLs are different from typical peptic ulcers because the mucosal defect in DLs is not surrounded by inflammatory cell infiltration, and the exposed artery has a large
Correspondence: Tonguç Utku Yılmaz Acıbadem University, Atakent Hospital, Organ Transplantation Unit, Turgut Özal Tel +90 2I2 404 444।

Email tonyutku@hotmail.com 
diameter. The surrounding mucosa is normal. There are no aneurismal, arteriosclerotic, or vasculitis changes in these dilated, tortuous, submucosal arteries. A locally protruding blood vessel in the submucosal layer causes mucosal blood circulation disturbances, and the stress load (such as erosion or atrophy) causes a reduction in blood flow in the overlying mucosa. ${ }^{2}$ Previously published cases involving affected newborns describe lesions that are congenital in nature. ${ }^{3}$ Most patients with DL have a history of non-steroidal antiinflammatory drug, aspirin, and/or warfarin use or comorbid diseases such as cardiopulmonary and renal diseases and/or diabetes that may contribute additional stress factors. ${ }^{4,5}$ Solid bowel content can contribute to mucosal stercoral ulceration in the colon, but there is no specific pathogenesis for duodenal DL. Senile atrophy is another factor involved in DL rupture and hemorrhage. Studies on the history of comorbid diseases have described that most patients with duodenal and jejunal DLs have a history of drug abuse, rheumatic fever, congestive heart failure, hypertension, cirrhosis, diabetes, permanent pacemaker, aortic stenosis, cerebral vascular accident, obstructive sleep apnea, chronic obstructive pulmonary disease, hyperlipidemia, coronary artery disease, atrial fibrillation, renal failure, and/or pulmonary hypertension. ${ }^{6-9}$ Norton et al showed that duodenal DLs constituted $18 \%$ of all DLs in their study, and although not classified according to the organs, the total comorbidity rate was $90 \% .{ }^{10}$ The patients in 3 studies did not present with any previous health problems. ${ }^{11-13}$ The medical history of adult patients was not mentioned in the study conducted by McClave et al ${ }^{14}$ Patients with duodenal and jejunal DLs without any comorbid diseases were usually pediatric patients. ${ }^{3}$ Concomitant peptic ulcer disease was reported in $11 \%$ of patients with duodenal DLs. ${ }^{10}$ This might be the reason for erosion of the overlying mucosa. These results suggest that the mucosa overlying the congenital tortuous submucosal arteries is somewhat eroded by systemic or local effects in conjunction with bleeding. Masked silent anomalies in the duodenum were observed less often than in the stomach because the duodenal mucosa is less vulnerable than the gastric mucosa to this congenital condition. The adverse effects of hyperacidity on duodenal mucosa are known, and it is more susceptible to erosion because of its enzymatic content and acid exposure. Duodenal ulcers are seen 3-4 times more often than gastric ulcers, and ulcer complications are also present more often in the duodenum.

Because recent treatment modalities for DL patients use the endoscopic approach, histological/pathological results were only obtained in older studies. In the hisological/pathological examinations of duodenal DL, the arterial wall consists of the intima, media, and adventitia. Thus, Dieulafoy's lesion was not an aneurysm. Arteries appeared healthy and showed no evidence of vasculitis or atheromatous disease. There was no arteriovenous malformation in the pathological examinations. ${ }^{13}$ The mean age of adult patients with duodenal DL data that were retrievable was 67.08 years (range $21-86$ years). ${ }^{6-14}$ The mean age of patients with duodenal DLs in the above-mentioned studies was similar to patients with DL in all localizations (mean age, 61 years; range, 7-72 years). ${ }^{6}$ The mean age of patients with jejunal lesions was 67.4 years (range 21-86 years) in previously published studies. ${ }^{8-13}$ There was no sex predominance in jejunal DL. ${ }^{8}$ Similar to DL, patients with jejunal DL were using anticoagulant treatment and had several comorbid diseases. In agreement with previous studies and in contrast to male predominance in gastric DL, there was no male predominance in duodenal and/or jejunal DL., ${ }^{5,6}$ This was probably because of the protective role of estrogen on the gastric mucosa and preservation of the mucosa overlying the DL in stomach. Estrogen protects the stomach by increasing prostaglandin synthesis, which reduces gastric acid and pepsin. Prostaglandins increase exocrine pancreatic secretion, and positive effects in the small intestine are limited by increasing mucous secretion.

\section{Clinical presentation and diagnosis}

The clinical presentation of DLs is painless with massive periodic recurrent intermittent hematemesis associated with melena, hematochezia, and hypotension. Concomitant peptic ulcer or gastritis might present with previous dyspeptic symptoms. While duodenal DL patients mostly present with hematemesis, jejunal DL patients mostly present with melena. Hemodynamic stability is important in making a diagnosis and deciding upon treatment modalities. The clinical DL findings are similar to GI bleeding and include hematochezia, melena, hematemesis, tachycardia, hypotension, and syncope. Intermittent and massive GI bleeding is included in the classical DL presentation. Patients presented with melena (44\%), hematemesis (30\%), melena and hematemesis (18\%), and hematochezia and iron-deficiency anemia $(6 \%))^{3,5}$ The mean blood hemoglobin levels in patients presenting with DL ranged from 4 to $14 \mathrm{~g} / \mathrm{dL} .^{3-8}$ The clinical presentations of patients with DL depends on the duration of bleeding, general condition of the patient, localization of bleeding, and diameter of the bleeding vessel. While patients with duodenal lesions generally present with symptoms of upper GI bleeding, patients with jejunal DL present with symtoms of lower GI bleeding (Table 1). 
Table I Age, sex, presenting symptoms, and average number of endoscopies needed prior to diagnosis for both duodenal and jejunal DLs

\begin{tabular}{|c|c|c|}
\hline Demographic data & Duodenal DL & Jejunal DL \\
\hline Mean age (years), (range) & $67.08(21-86)$ & $67.4(21-86)$ \\
\hline Sex predominance & No & No \\
\hline Most common presentation & Hematemesis & Melena \\
\hline $\begin{array}{l}\text { Mean average number of endoscopies } \\
\text { for diagnosis (range) }\end{array}$ & I.8 (I-8) & $\mathrm{I} .5(\mathrm{I}-3)$ \\
\hline $\begin{array}{l}\text { Rate of re-bleeding after endoscopic } \\
\text { treatment (\%) }\end{array}$ & $0-10$ & $12.5-20$ \\
\hline
\end{tabular}

Abbreviation: DL, Dieulafoy's lesion.

In the past, the diagnosis was rarely made before surgery or postmortem examination. The diagnosis could only be made by histological/pathological examination. However, with increased surgical experience, the diagnostic criteria are now dependent on endoscopic findings. ${ }^{15}$ Endoscopic criteria for DLs include several parameters: 1) active arterial spurting or micropulsative streaming from a tiny mucosal defect or through the normal surrounding mucosa; 2) visualization of a protruding vessel with or without active bleeding within a tiny mucosal defect or through the normal surrounding mucosa; and/or 3) fresh, densely adherent clot(s) with a narrow point of attachment to a tiny mucosal defect or to normal appearing mucosa. ${ }^{15}$ Currently, DL is diagnosed endoscopically rather than histologically. Upper GI endoscopy can easily reach the duodenum and help to diagnose and treat DL. However, the diagnosis may be difficult to make because of the small nature of the lesion, the normal appearance of the surrounding mucosa, and the intermittent nature of the hemorrhages. Therefore, multiple endoscopies are often necessary to make the diagnosis. ${ }^{6,11,16}$ In addition to the nature of the lesion, massive hemorrhage in the limited lumen of the duodenum can be overlooked because of other concomitant lesions such as an ulcer, and an inexperienced endoscopist who looks for an inflammed lesion can overlook the DL because of clots, duodenal angulations, or periampulary diverticulum. Thus, the duodenal DL cannot always be controlled on the first attempt. Lopez-Arce et al showed that patients underwent at least 3 attempts to control the duodenal DL bleedings. ${ }^{16}$ To overcome this problem, early endoscopic evaluation and side-viewing endoscopy is needed. ${ }^{17,18}$ Endoscopies performed within the first 12 hours have a high success rate for diagnosing DLs because of their capability to pinpoint the bleed location. Duodenal DLs located in the periampullary area and in the second portion of the duodenum cannot be seen or treated by forward-viewing endoscopies. ${ }^{12,17}$ Barium studies were unsuccessful in duodenal DL diagnosis.
Jejunal DLs are very rare and constitute only $3.5 \%$ of all small intestinal bleeds. ${ }^{19}$ There were $<50$ patients with jejunal DLs in the literature. ${ }^{8}$ Jejunal DLs are not in the range of standard endoscopies, except the proximal ones. Thus, push, single-, or double-balloon enteroscopy can be used for both diagnosis and treatment. Alternatively, capsule endoscopy, angiography, and red cell scanning can be used to pinpoint the location of the jejunal DL. Angiography and red cell scanning can be used to determine the location of the bleed. There is no specific radiological view of the lesion. The angiographic findings include extravasation of contrast from an eroded artery that may appear normal. However, existence of a tortuous and ectatic artery in angiography can provide important information for the diagnosis of DL. ${ }^{5}$ Although the patients' hemodynamic status is important for deciding on diagnostic modalities, most of the patients were evaluated first by colonoscopy or endoscopy for the evaluation of GI bleeding. Technetium scintography was used in 2 studies but it was unable to show any bleeding site. ${ }^{20,21}$ Angiography can show bleeding points that originate from the mesenteric arteries. If standard angiography fails, formal heparin therapy with an angiogram can be used to show the exact bleeding point. The selective placement of an angiographic catheter into the affected artery may also have resuscitative and possible therapeutic benefits. Intra-arterial vasopressin installation or embolization may aid in slowing any hemorrhaging, thereby allowing more time for further resuscitative efforts. Patients who undergo embolization are at risk of ischemia of the non-involved jejunal segments unless the cathether has been placed distal to the subsegmental branches. Angiographic embolization is the choice of treatment in suitable patients. Angiography failed to show the bleeding point in 2 of 4 jejunal DL patients, and embolization was also unsuccessful in these patients. ${ }^{8,13,21,22}$ The most likely reason was the relative mobility of the jejunum and the thinness of its wall, both of which made it difficult to obtain the desired compression of the vessel by angiographic embolization. Patients who underwent successful embolization for the treatment of DL were also shown to have another DL in another organ. ${ }^{23}$ Capsule endoscopy is a frequently used method to evaluate bleeding in the small intestine. It is a method of choice for small intestine imaging after colonoscopy and endoscopy for mid-GI bleeding in hemodynamically stable patients. ${ }^{19}$ Capsule endoscopy has pinpointed the origin of the bleeding in all patients with jejunal DL. ${ }^{8,19,20,22}$ Localization of the bleeding point guides the clinician in the best way to approach the lesion. The decision as to whether the initial enteroscopy was performed from an oral or anal approach was based 
primarily on clinical presentation and the result of capsule endoscopy. If capsule endoscopy reveals a possible bleeding source within the proximal two-thirds of the small bowel, an oral route enteroscopy is performed first because it can reach the bleeding point. If no bleeding is detected along the oral route, a submucosal tattoo is placed to mark the deepest insertion point and the anal route is then used. Tattooing can also be used for marking the lesion(s) during surgery when endoscopic treatments fail. Tattooing the bleeding site with India ink maybe helpful for future identification in the event of recurrent bleeding. Single- or double-balloon enteroscopy is used to treat mid-GI bleeds. Chronic intermittent bleeding may be encountered when treating jejunal DL, which results in multiple balloon-assisted enteroscope procedures before diagnosis; it was reported that at least 2 or more enteroscope procedures were needed before diagnosis. ${ }^{19,24}$ Although Dulic-Lakovic et al reported that 1.5 endoscopies were required per patient to diagnose jejunal DL, Lipka et al reported that all jejunal DL patients were diagnosed after the first attempt. ${ }^{8,19}$ Single- or double-balloon enteroscopy is mostly preferred method for treatment of jejunal DLs in recent studies. If the patient is hemodynamically unstable or if technical experience with endoscopic treatment is limited, surgical evaluation is needed. Intraoperative enteroscopy from an enterotomy can help to focus on the area containing the bleed. ${ }^{13,21}$ Lipka et al also showed that DLs were localized in the proximal jejunum. ${ }^{8}$ Dulic-Lakovic et al showed that the mean distance of the jejunal DL from the pylorus was $132 \pm 115 \mathrm{~cm} .{ }^{19}$ For both duodenal and jejunal DLs, DLs in other accompanying organs should be considered. ${ }^{22}$

\section{Treatment}

DLs are a cause of GI bleeding, and treatment depends on the presentation, lesion site, and available surgical and endoscopic expertise. Bleeding from DLs may be life threatening and they were treated surgically before 1990. Since then, endoscopic treatment has replaced surgery as the treatment of choice. Several endoscopic treatment methods, including injection, a heater probe, bipolar electrocoagulation, and laser therapy have been used. Since then, mechanical devices such as hemoclips and rubber band ligation have been presented as new choices for the treatment of GI bleedings.

Injection therapies mainly aim to stop bleeding from vessels by injection of several agents such as vasoconstrictors (epinephrine) or sclerosants (ethanol, aethoxysklerol, or polidocanol). ${ }^{17}$ One DL patient who was treated with N-butyl 2 cyanoacrylate has been described in the literature. ${ }^{25}$ Epinephrine can be injected (dilution of 1:10,000) into 4 quadrants, with an initial dose of $2.5 \mathrm{~mL}$ that can be repeated. This is an inexpensive, simple, and relatively safe procedure. There is the risk, however, of re-bleeding. In 1 case, epinephrine was used as the sole therapy in duodenal DL without re-bleeding. However, it has been shown that epinephrine plus a heat probe is the most preferred treatment method in duodenal DL. ${ }^{6}$ Initial injection with epinephrine, followed by other treatment methods, seems like an optimal treatment for acute bleeding because it provides the advantages of temporary hemostasis without risk of tissue injury and perforation. Achieving enhanced visualization of the bleeding site should guide the second definitive treatment method (such as a combination of mechanical approaches) and reduce the risk of complications.

Thermal endoscopic treatments stop the bleeding using heat either by contact or non-contact. Bipolar and heater probes are examples of contact thermocoagulation, and argon is an example of a non-contact method. Contact thermocoagulators carry a risk of transmural injury in thin walled organs such as the duodenum. The non-contact methods have less tissue depth injury, and they are safer than the contact methods. However, they are only effective for superficial coagulation. There is no reported case of perforation after duodenal DL treatment with a heater probe. Schmulewitz et al presented 3 cases in which duodenal DLs were treated only with heater probes. ${ }^{6}$

Mechanical hemostasis is performed using hemoclips and band ligation. Hemoclips have been suggested to be more successful than thermal ablation in achieving hemostasis for bleeding DLs. The success rate using hemoclips is generally high when the surrounding tissue is soft. Thus, hemoclips are advantageous for hemostastis in DLs because the size of the lesion is small, and hemostasis can be obtained by concurrently clipping the normal surrounding mucosa. It is difficult, however, to apply hemoclips in difficult locations such as the duodenum; acute duodenal angulation makes hemoclip application maneuvers impossible. Side-view endoscopes, which require surgical expertise, may be needed for hemoclip application in difficult locations. ${ }^{12}$ Hemoclips that were previously placed incorrectly can hinder accurate positioning of subsequent hemoclips. Thus, it is important to apply the hemoclips accurately to the DL the first time they are used. Epinephrine injection(s) before hemoclip application might be helpful in facilitating this procedure.

Band ligation is easier than hemoclip application because of its accessibility to difficult locations. It is safer than thermal techniques in terms of minimizing perforations. There is a risk, however, of re-bleeding ulcer formation around the ligated mucosa. Another problem is the required time interval 
for preparing the overtube and other pieces of equipment for band ligation. Chung et al compared the efficacy of hemostatic methods in patients with DL, and they reported that mechanical methods for hemostasis such as band ligation and hemoclipping were superior to injection methods for controlling bleeding and preventing recurrent bleeding. ${ }^{26}$ In duodenal DLs, however, mechanical techniques are difficult and injection plus thermal ablation achieves higher success rates with less re-bleeding.

Based on these advantages of endoscopic treatment, nearly all patients with DLs can be treated with endoscopic methods; primary hemostasis may occur in more than $90 \%$ of patients. Re-bleeding has been found and has not been associated with age, sex, medical history, or location. ${ }^{27}$ Duodenal localization does not appear to present a risk factor for re-bleeding. Monotherapy with adrenaline and arterial spurting are risk factors for re-bleeding. For this reason, combined therapy is advised for duodenual DLs. Park et al showed that re-bleeding was not associated with DL location or treatment method. ${ }^{28}$ The presence of infection and kidney disease were related to re-bleeding.

If endoscopic equipment is lacking or endoscopic treatment of duodenal DLs has failed, exploratory surgery must be performed. The rate of surgical necessity for DLs has decreased from $100 \%$ to $3 \%$ with increasing surgical experience. If the localization of the duodenal DL is known, it is preferable to longitudinally open that part of the duodenum. If the location is not known because there is no predominant localization of the duodenal DL, it would be better to longitudinally open the anterior wall of duodenal bulb. This would help the surgeon evaluate the papilla, any possible ulcers, and the distal part of the duodenum. The bleeding point can be overlaid with a 3-point $U$ stitch technique. Care must be taken to avoid incorporating the common bile duct into a stitch. The course of the comon bile duct can be identified by inserting a probe through the ampulla. After bleeding is controlled, the duodenotomy should be closed transversely to avoid narrowing. Unless pylorotomy is perfomed, there is no need for vagotomy. If the source of bleeding cannot be defined, emergent pancreaticoduodenectomy should be perfomed. $^{29}$

Patients with DL usually present with GI bleeding. Therefore, the patients should first be treated using a general GI bleeding approach. In these cases, necessary fluid replacement, blood transfusions, and blood products, and close follow-up monitoring should be provided. The main factor in deciding which treatment modality to use is patient's hemodynamic status. If the patient is hemodynamically unstable, exploratory surgery must be performed. However, without localization of the bleeding point, exact resection of the jejunal DL cannot be perfomed on the first attempt. ${ }^{21}$ Because DLs do not have any fibrosis, inflammation, or surrounding polyps, it is nearly impossible to feel the DL. For this reason, intraoperative endoscopy (oral, anal, or via enterotomy) is needed (Figure 1). ${ }^{13,30}$ Historically, intraoperative methylene blue injection via the selectively positioned catheter can be used to identify the affected jejunal segment. Segmental resection and anastomosis is the preferred surgical choice to treat jejunal DL (Figure 2). ${ }^{13,19,21}$ Simple overlaying of the lesion is not recommended because it is associated with a greater risk of recurrent bleeding. There was no re-bleeding in surgically treated patients. Jejunal DL bleeding is generally obscure, and early treatment is needed. The primary modality of endoscopic treatment is electrocautery and multipolar electrocoagulation. Epinephrine injection is used as an adjuvant therapy to initially slow down the bleeding. If coagulation is unsuccessful, clip placement is used for hemostasis. Argon plasma coagulation is the first choice to treat patients with

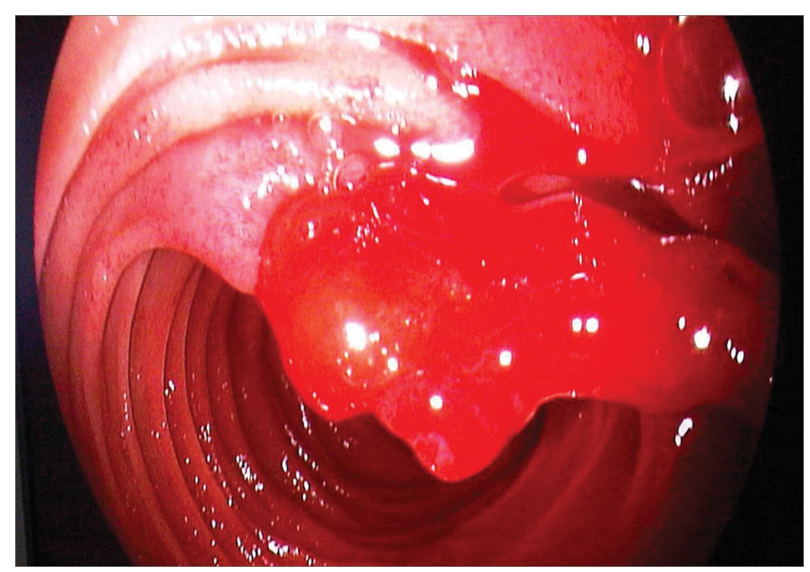

Figure I Endoscopic view of jejunal Dieulafoy's lesion.

Notes: Visualization of the protruding vessel with active bleeding within a tiny mucosal defect or through the normal surrounding mucosa.

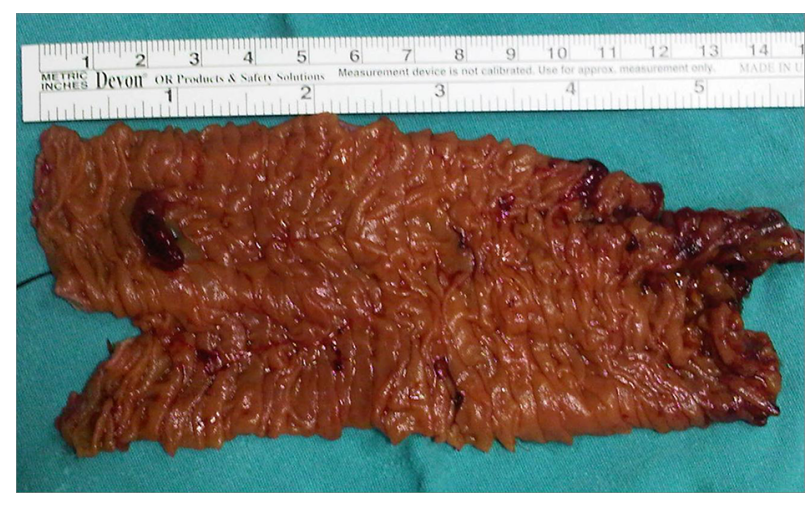

Figure $\mathbf{2}$ Resection material from the jejunum that includes a Dieulafoy's lesion. 


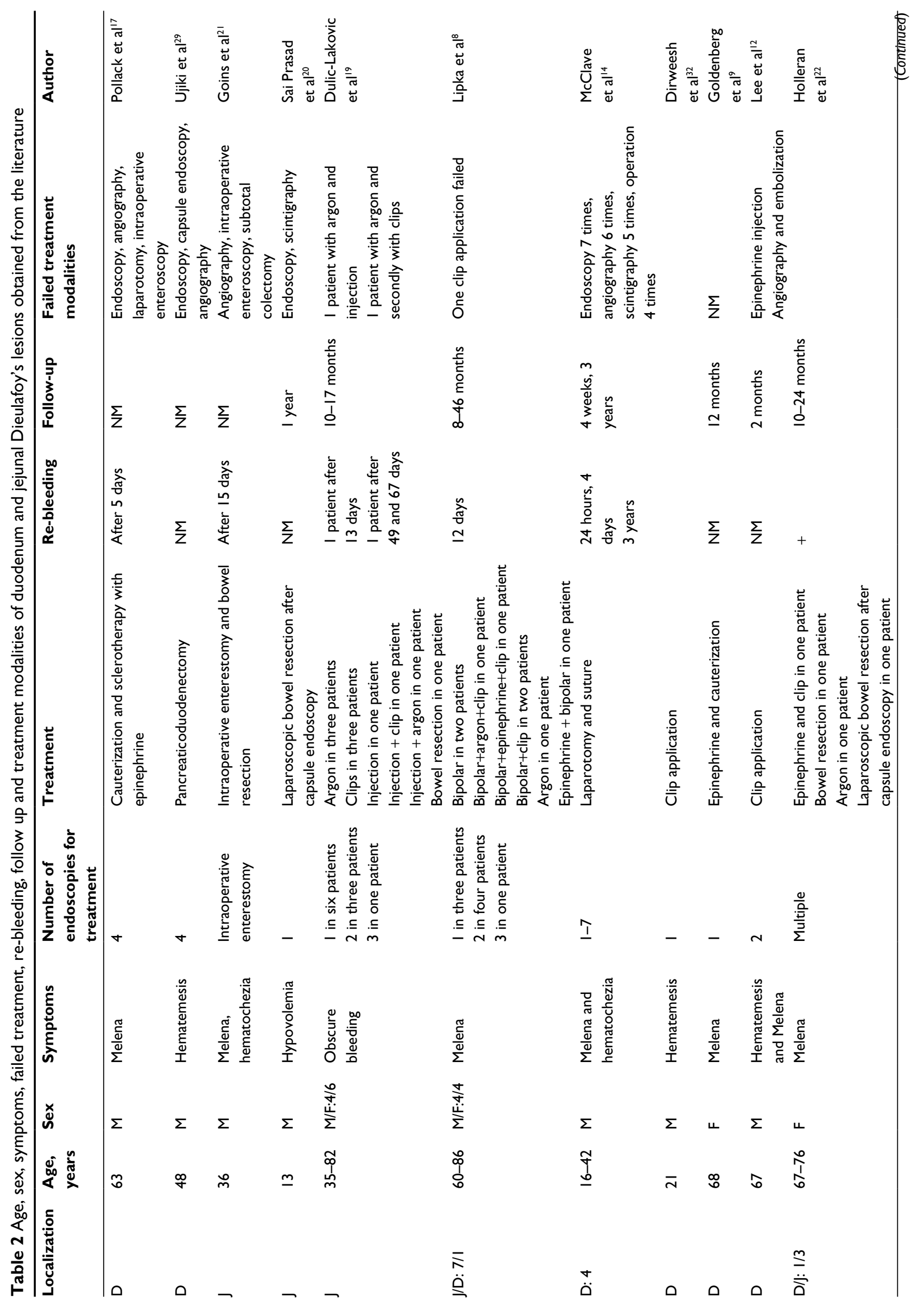




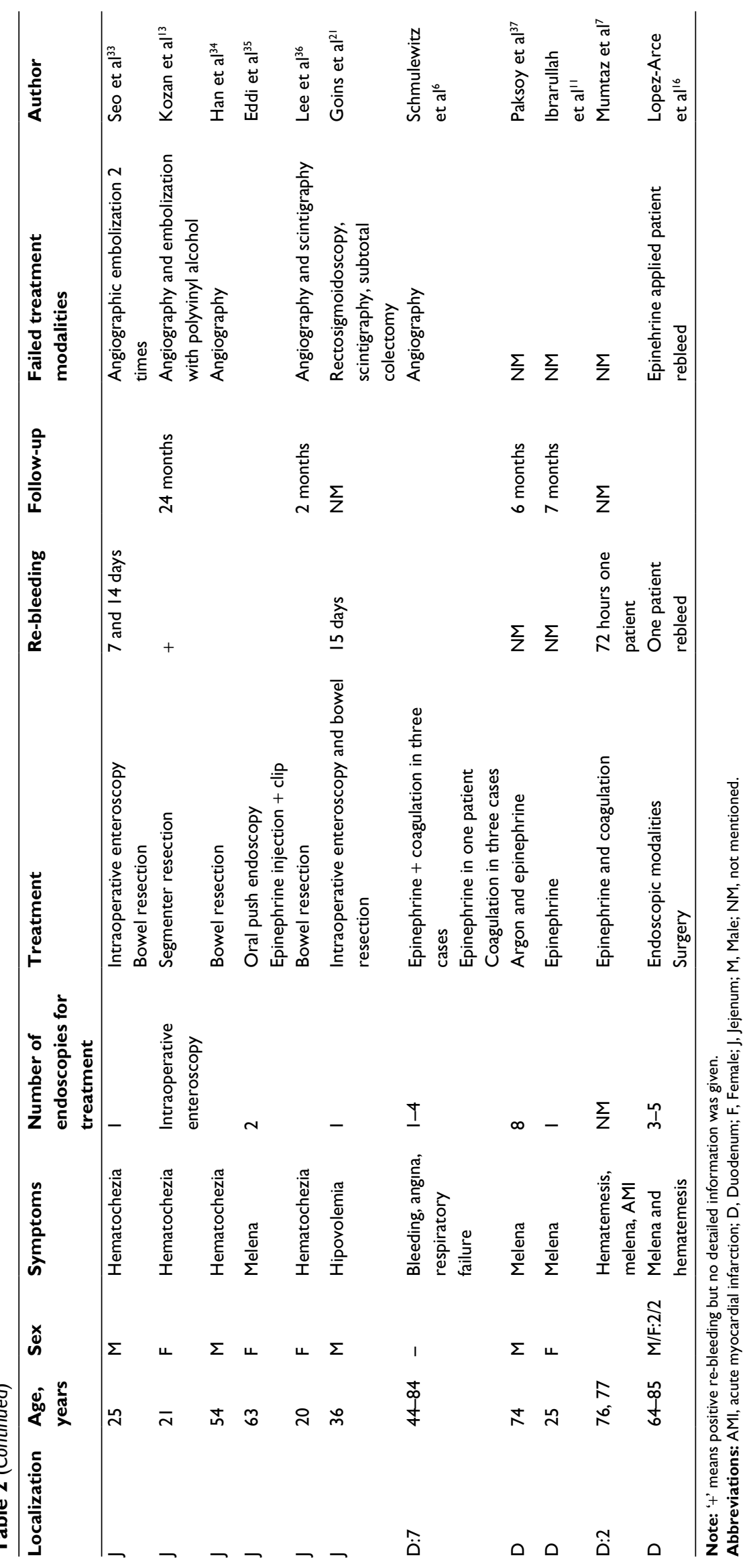


jejunal DLs. ${ }^{8}$ There were 4 reported cases of re-bleeding in jejunal DL patients after endoscopic treatment. The rate of re-bleeding after endoscopic treatment of jejunal DL is given as $12.5 \%$ and $20 \%$ in previous studies. ${ }^{8,19,22}$ The re-bleeding times were 12, 13, and 49 days and 12 weeks after the first treatment. ${ }^{8,19,22}$ The primary hemostasis treatments were argon and clips. There is not enough data to permit comparison of the treatment modalities. Studies have shown that mechanical endoscopic methods such as hemoclip and band ligation are more effective than injection and thermal therapy for general DL. ${ }^{25}$ Additionally, it has been shown that using a combination of 2 endoscopic techniques for hemostasis is superior to using a single method for treating gastroduodenal ulcer disease. Therefore, optimal endoscopic treatment of jejunal DL would be to combine thermal therapy and clip application. Mortality after jejunal DL bleeding depends on existing comorbid diseases such as cardiovascular diseases or cirrhosis. For this reason, early diagnosis and treatment is important. The mortality rates described in previous studies, in which endoscopic treatment was not popular, were between $23 \%$ and $35 \%$. With increased surgical experience in endoscopic treatment and awareness of DL, early diagnosis has decreased mortality rates by up to $8 \%{ }^{5}$ While the mortality rates in previous studies were associated with massive bleeding, recent mortality rates were mostly associated with comorbid diseases such as heart and/or respiratory failure, sepsis, and/or cirrhosis. ${ }^{7,22,28}$

When re-bleeding occurs, diagnostic and treatment approaches were the same as the previous treatment approaches. As there is a risk of re-bleeding in DL patients even 12 weeks after the initial treatment, the clinician should follow-up with the patient for at least 6 months. A longer follow-up does not add any benefit for protection against re-bleeding. ${ }^{31}$ In addition to the re-bleeding risk, there is a risk of overlooked DL in another part of the GI system. Risk of overlooked DL increases in the presence of ulcers. The demographic information, treatment methods, failed methods, re-bleeding rates, follow-up data obtained from the previous studies are given in Table 2.

There are several treatment algorithms for DLs that are presented in the literature. As a general rule, initial resuscitation is universal for all types of bleeding from DLs. Differences depend on the availability and experience at the centers. Although minimally invasive techniques are currently gaining more importance, the best way is to treat the patient with DL bleeding should depend on the availabilities of the health center. Early intervention and a cautious approach is the most important step in DL diagnosis and treatment.

\section{Conclusion}

DLs are rare, but it is important to be aware of this condition because DLs can cause massive hemorrhaging that can be life threatening. The location of the lesion and the hemodynamic status of the patient are the most important factors in the diagnosis and treatment modalities. It is preferable to use endoscopic methods for diagnosis in patients who are hemodynamically stable. In addition to diagnosis, multiple endoscopic treatments can be performed concurrently or in combination. To decrease the number of endoscopic sessions needed to achieve hemostasis, it is important to select the best treatment options and be aware of possible DLs when evaluating a patient who has GI bleeding. Surgical treatment is advantageous with low risk of re-bleeding, and surgery should be selected when patients are hemodynamically unstable and when other methods have failed. Additionally, the facilities and surgical experience of the centers to which patients are referred have a clear role in determining the selected treatments in their approach to treating DL patients. In conclusion, we recommend that clinicians develop a patient-specific strategy for DL patients, which includes consideration of the facilities at the centers in which they work.

\section{Disclosure}

The authors report no conflicts of interest in this work.

\section{References}

1. Veldhuyzen Van Zanten SJO, Bertelsman JFWM, Schipper MEI, Tytgat GNJ. Recurrent massive hematemesis from Dieulafoy vascular malformation: a review of 101 cases. Gut. 1986;27(2):213-222.

2. Miko TL, Thomazy VA. The caliber persistent artery of the stomach: a unifying approach to gastric aneurysm, Dieulafoy's lesion, and submucosal arterial malformation. Hum Pathol. 1988;19(8):914-921.

3. Senger JL, Kanthan R. The evolution of Dieulafoy's lesions since 1897: then and now - A journay through the lens of a pediatric lesion with literature review. Gastroenterol Res Pract. 2012;2012:432517.

4. Lee YT, Walmsley RS, Leong RW, Sung JJ. Dieulafoy's lesion. Gastrointest Endosc. 2003;58(2):236-243.

5. Baxter M, Aly EH. Dieulafoy's lesions: current trends in the diagnosis and management. Ann R Coll Surg Engl. 2010;92(7):548-554.

6. Schmulewitz N, Baillie J. Dieulafoy lesions: a review of 6 years of experience at a tertiary referral center. Am J Gastroenterol. 2001;96(6): $1688-1694$.

7. Mumtaz R, Shaukat M, Ramirez FC. Outcomes of endoscopic treatment of gastroduodenal Dieulafoy's lesion with rubber band ligation and thermal/injection therapy. J Clin Gastroenterol. 2003;36(4):310-314.

8. Lipka S, Rabbanifard R, Kumar A, Brady P. A single-center United States exerience with bleeding Dieulafoy lesion of the small bowel: diagnosis and treatment with single-balloon enteroscopy. Endosc Int Open. 2015;3(4):E339-E345.

9. Goldenberg SP, DeLuca VA Jr, Marignani P. Endoscopic treatment of Dieulafoy's lesion of the duodenum. Am J Gastroenterol. 1990;85(4):452-454.

10. Norton ID, Petersen BT, Sorbi D, Balm RK, Alexander GL, Gostout CJ. Management and long term prognosis of Dieulafoy lesion. Gastrointest Endosc. 1999;50(6):762-767. 
11. Ibrarullah M, Wagholikar GD. Dieulafoy's lesion of duodenum: a case report. BMC Gastroenterol. 2003;3:1-5.

12. Lee WS, Cho SB, Park SY, et al. Succesful side-viewing endoscopic hemoclipping for Dieulafoy-like lesion at the brim of a periampullary diverticulum. BMC Gastroenterol. 2010;10:24-29.

13. Kozan R, Gülen M, Yılmaz TU, Leventoğlu S, Yılmaz E. Massive lower gastrointestinal bleeding from a jejunal Dieulafoy lesion. Ulus Cerrahi Derg. 2014;30(4):225-227.

14. McClave SA, Goldschmid S, Cunningham JT, Boyd WP Jr. Dieulafoy's cirsoid aneurysm of the duodenum. Dig Dis Sci. 1988;33(7):801-805.

15. Stark ME, Gostout CJ, Balm RK. Clinical features and endoscopic management of Dieulafoy's disease. Gastrointest Endosc. 1992;38(5):545-550.

16. Lopez-Arce G, Zepeda-Gomez S, Chavez-Tapia NC, et al. Upper gastrointestinal Dieulafoy's lesions and endoscopic treatment: first report from a Mexican centre. Therap Adv Gastroenterol. 2008;1(2):97-101.

17. Pollack R, Lipsky H, Goldberg RI. Duodenal Dieulafoy's lesion. Gastrointest Endosc. 1993;39(6):820-822.

18. Lara LF, Sreenarasimhaiah J, Tang SJ, Afonso BB, Rockey DC. Dieulafoy lesion of the GI tract: localization and therapeutic outcomes. Dig Dis Sci. 2010;55(12):3436-3441.

19. Dulic-Lakovic E, Dulic M, Hubner D, et al. Bleeding Dieulafoy lesions of the small bowel: a systematic study on the epidemiology and efficacy of enteroscopic treatment. Gastrointest Endosc. 2011;74(3):573-580.

20. Sai Prasad TR, Lim HL, Lim KH, Yap TL. Bleeding jejunal dieulafoy pseudopolyp: capsule endoscopic and laparoscopic-assisted resection. J Laparoendosc Adv Surg Tech A. 2007;17(4):509-512.

21. Goins WA, Chatman DM, Kaviani MJ. Massive lower gastrointestinal bleeding due to 'Dieulafoy's vascular malformation' of the jejunum: case report. J Natl Med Assoc. 1995;87(10):766-770.

22. Holleran G, Hussey M, McNamara D. Small bowel Dieulafoy lesions: an uncommon cause of obscure bleeding in cirrhosis. World J Gastrointest Endosc. 2016;8(16):568-571.

23. Reilly HF 3rd, al-Kawas FH. Dieulafoy lesion. Diagnosis and management. Dig Dis Sci. 1991;36(12):1702-1707.

24. Landaeta J, Paternina R. Efficacy of endoscopic treatment of Dieulafoy lesions of the small bowel. In: Proceedings from the Digestive Disease Week Annual Meeting; 2013;May 13-21:Abstract SA1665 Orlando 201.
25. D'Imperio D, Papadia C, Baroncini D, Piemontese A, Billi P. N-butyl 2 cyanoacrylate in the endoscopic treatment of Dieulafoy ulcer [Abstract]. Endoscopy. 1995;27(2):216.

26. Chung IK, Kim EJ, Lee MS, et al. Bleeding Dieulafoy's lesions and the choice of endoscopic method: comparing the hemostatic efficacy of mechanical and injection methods. Gastrointest Endosc. 2000;52(6): 721-724.

27. Jamanca-Poma Y, Velasco-Guardado A, Pinero-Perz C, et al. Prognostic factors for recurrence of gastrointestinal bleeding due to Dieulafoy's lesion. World J Gastroenterol. 2012;18(40):5734-5738.

28. Park SH, Lee DH, Park CH, et al. Predictors of rebleeding in upper gastrointestinal Dieulafoy lesions. Clin Endosc. 2015;48(5):385-391.

29. Ujiki MB, Swanstrom LL, Diwan TS, Hansen PD. Emergent pancreaticoduodenectomy for Dieulafoy lesion of the duodenum. Am Surg. 2010;76(6):656-657

30. Blecker D, Bansal M, Zimmerman RL, et al. Dieulafoy's lesion of the small bowel causing massive gastrointestinal bleeding: two cases reports and literature review. Am J Gastroenterol. 2001;96(3):902-905.

31. Baettig B, Haecki W, Lamm-er F, Jost R. Dieulafoy's disease: endoscopic treatment and follow up. Gut. 1993;34:1418-1421.

32. Dirweesh A, Chikezie A, Khan MY, Zia S, Tahir M. Postural syncope and constipation: an unusual presentation of a duodenal Dieulafoy's lesion. Case Rep Gastrointest Med. 2017;2017:6983434

33. Seo KI, Moon W, Lee CW, et al. Minimal resection of Jejuna Dieulafoy's lesion using an intraoperative fluoroscopic localization of the metallic coils used in angiography. Korean J Gastroenterol. 2017;69(2):135-138.

34. Han MS, Park BK, Lee SH, Yang HC, Hong YK, Choi YJ. A case of Dieulafoy lesion of the jejunum presented with massive hemorrhage. Korean J Gastroenterol. 2013;61(5):279-281.

35. Eddi R, Shah N, Depasquale JR. Gastrointestinal bleeding due to a Dieulafoy lesion in the afferent limb of a billroth II reconstruction. Gastroenterol Hepatol (NY). 2011;7(4):268-271.

36. Lee KS, Moon YJ, Lee SI, et al. A case of bleeding from the Dieulafoy lesion of the jejunum. Yonsei Med J. 1997;38(4):240-244.

37. Paksoy F, Bes C, Aniktar H, Karaca Ç, Borlu F. Dieulafoy lezyonu: Üst gastrointestinal sistem kanamaların nadir bir nedeni: Üç olgu sunumu [Dieulafoy's lesion. A rare cause of uppergastrointestinal system bleeding. Three cases]. Akademik Gastroenteroloji dergisi. 2009:8(1):35-37.

\section{Clinical and Experimental Gastroenterology}

\section{Publish your work in this journal}

Clinical and Experimental Gastroenterology is an international, peerreviewed, open access, online journal publishing original research, reports, editorials, reviews and commentaries on all aspects of gastroenterology in the clinic and laboratory. This journal is included on PubMed. The manuscript management system is completely online

\section{Dovepress}

and includes a very quick and fair peer-review system, which is all easy to use. Visit http://www.dovepress.com/testimonials.php to read real quotes from published authors. 